\title{
Sopra una delle esperienze del Plateau.
}

\author{
(Di E. Almaysi, a Genova.)
}

1. Il Platrau, in una delle sue celebri esperienze, esamina le configurazioni d'equilibrio di una goccia d'olio, immersa in un liquido di ugual densità (acqua ed alcool, in proporzioni convenienti) e appoggiata a dei sostegni rigidi fissi.

Sia $W$ il potenziale delle forze agenti sull'intero sistema (forze di gravità e forze capillari). Condizione necessaria e sufficiente per l'equilibrio sarà che per ogni deformazione infinitesima della massa liquida, il differenziale di $W$ sia nullo o positivo.

Se per una data configurazione d'equilibrio $W$ è minimo, l'equilibrio ̀̀ stabile.

Limitando il nostro studio alle configurazioni d'equilibrio della goccia d'olio, possiamo supporre di mantenere inalterata la superficie libera dell'intera massa liquida. Allora quella parte di $W$ che è dovuta alle forze di gravità si mantiene costante: potremo quindi considerare $W$ come il potenziale delle sole forze capillari. Esso è dato dalla formula:

$$
W=m o-n \tau
$$

ove $\sigma$ e $\tau$ denotano, rispettivamente, le superficie di separazione della goccia d'olio col liquido circostante, e coi sostegni : $m$ ed $n$ sono delle costanti. La costante $m$ è positiva.

In particolare il Prateau esamina il caso che i sostegni siano costituiti da due dischi circolari, di ugual raggio, orizzontali, e con i loro centri situati sopra una retta verticale.

Diciamo $H$ la distanza fra i due dischi, $R$ il loro raggio.

Una configurazione d'equilibrio si presenta quando la goccia d'olio abbia la forma di un cilindro di raggio $R$, colle basi a contatto coi dischi. 
In tal caso il Pratead ha osservato che l'equilibrio è síabile o instabile secondochè $H$ e minore o maggiore di $2 \pi R$.

Questo fatto, constatato sperimentalmente, $\grave{e}$ in relazione col seguente Teorema :

"L'area di una superficie cilindrica $\sigma$, limitata da due circonferenze di raggio $R$, situate in piani normali alla retta che congiunge $\mathrm{i}$ loro centri, ̀̀ minima rispetto all'area di tutte le superficie $\sigma^{\prime}$, infinitamente vicine, che passano per le stesse circonferenze, e racchiudon', insieme ai cerchi di base, lo stesso volume, purchè la distanza $H$ fra $i$ due cerchi sia minore di $2 \pi R$. Se $H$ è maggiore di ' $2 \pi R$, esistono delle superficie $\sigma^{\prime}$ aventi un'area minore di $\sigma . \eta$

In questa Nota io do una dimostrazione del teorema enunciato. Dimostro poi un altro teorema, più generale. Valendomi di questo secondo teorema, faccio vedere che quando $H$ è minore di $2 \pi R$ la variazione del potenziale $W$ è positiva per qualunque deformazione infinitesima data alla goceia d'olio: mentre se $H$ è minore di $2 \pi R$, può esser negativa.

La questione di cui mi occupo era già stata esaminata dallo stesso Plateau, dal Mathieu e dal Poincaré, ma non, a mio parere, in modo esauriente. Il Poincaré (Capillarité; p. 102) si limita a considerare quelle deformazioni infinitesime per le quali varia la superficie di separazione $\sigma$ della colonna d'olio col liquido circostante, ma non la superficie di contatto $\tau$ della colonna d'olio coi sostegni. Con questa limitazione il potenziale $W$ assume la forma $m \sigma+$ cost., e la sua variazione, a meno del fattore positivo $m$, è uguale alla variazione di $\sigma$. Posta così la questione, è sufficiente a risolverla il teorema sopra enunciato. Di questo teorema il Pouncaré dà infatti una dimostrazione (affatto diversa da quella che io do in questa Nota). Ma col supporre invariabile la superficie $\tau$ si viene, in sostanza, ad introdurre un nuovo vincolo, cio che non apparisce giustificato.

Tenendo conto anche di quelle deformazioni per le quali varia la superficie $\tau$, si è condotti ad imporre dei limiti al valore della costante $n$ che figura nell'espressione di $W$ : essa deve esser compresa fra 0 ed $m$. Questa condizione porta como conseguenza che, in generale, se è possibile l'equilibrio di una colonna cilindrica, costituita di un certo liquido $L$, immersa in un liquido $L^{\prime}$, non è possibile, inversamente, l'equilibrio di una colonna costituita del liquido $L^{\prime}$, immersa nel liquido $L$. 
2. Diamo una dimostrazione del Teorema enunciato nel paragrafo precedente.

Sieno $A B, C D$ (Fig. I) le due circonferenze di raggio $R$ che limitano la superficie cilindrica $\sigma$. Consideriamo poi una superficie $\sigma^{\prime}$, infinit. ${ }^{\text {te }}$ vicina a $\sigma$, limitata dalle stesse circonferenze, e che racchiuda, come $\sigma$, un volume uguale a $\approx R^{2} H$ :

Possiamo supporre che $\sigma^{\prime}$ sia una superficie di rivoluzione, giacchè l'area di una tal superficie è minore, come osserva il PoIncaré, dell'area di qualunque altra superficie le cui sezioni parallele alle basi siano uguali alle sezioni corrispondenti della superficie di rivoluzione.

Una superficie $\sigma^{\prime}$ di rivoluzione possiamo individuarla in questo modo: detto $r$ il raggio della sezione di $\sigma^{\prime}$ che dista di $x$ dal cerchio $A B$, poniamo

$$
r^{2}=R^{2}(1+u)
$$

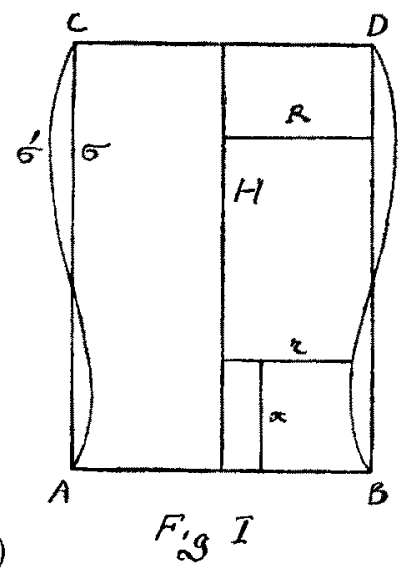

ove $u$ è una funzione della variabile $x$, definita fra $x=0$ ed $x=H$. Ad ogni funzione $u$ corrisponde una superficie $\sigma^{\prime}$.

La superficie $\sigma$ deve passare per i due circoli $A B, C D$; dunque per $x=0$ ed $x=H$ deve essere $r=R$, quindi $u=0$.

Inoltre la superficie $\sigma^{\prime}$ deve racchiudere insieme ai due cerchi un volume uguale a quello racchiuso dalla superficie $\sigma$, vale a dire deve essere:

$$
\int_{0}^{H} \pi r^{2} d x=\pi R^{2} H
$$

e sostituendo ad $r^{2}$ il suo valore

$$
\int_{0}^{H} u d x=0 .
$$

Noi supporremo che la $u$, oltre che della $x$, sia funzione di un parametro $t$, e che col tendere di $t$ a zero, $u$ tende uniformemente a zero.

Per la natura stessa del problema la $u$ deve essere una funzione continua della $x$. Ammetteremo che anche la sua derivata $\frac{d u}{d x}$ sia una funzione 
continua della $x$, e col tendere di $t$ a zero tenda uniformemente a zero. Questa limitazione è pienamente giustificata dal fatto che, a rigore, basterebbe (come fa il Polncará) confrontare la superficie a colle superficie di rivoluzione a curvatura costante: classe ben nota di superficie per le quali si riconosce facilmente che la condizione relativa a $\frac{d u}{d x}$ è soddisfatta. Però, la considerazione di questa sola classe di superficie $\sigma^{\prime}$ non apporterebbe nessuna semplificazione nei nostri calcoli.

Si tratta di dimostrare che quando $H$ è minore di $2 \pi R$, qualunque sia la funzione $u(x, t)$, purchè soddisfi le condizioni poste, si può trovare un numero $t_{0}$ tale che per ogni valore di $t$ compreso fra 0 e $t_{0}(0$ escluso) sia $\sigma^{\prime}>\sigma$; mentre se $H$ è maggiore di $2 \pi R, \sigma^{\prime}$ può conservarsi sempre minore di $\sigma$.

3. Cerchiamo un'espressione della superficie $\sigma^{\prime}$.

Detto $d l$ un elemento della linea $A C$ che rotando genera la superficie $\sigma^{\prime}$, avremo

$$
d l=\left\{1+\left(\frac{d r}{d x}\right)^{2}\right\}^{\frac{1}{2}} d x
$$

quindi :

$$
\begin{gathered}
\sigma^{\prime}=\int_{0}^{H} 2 \pi r d l=2 \pi \int_{0}^{H} r\left\{1+\left(\frac{d r}{d x}\right)^{2}\right\}^{\frac{1}{2}} d x= \\
=2 \pi \int_{0}^{H}\left\{r^{2}+\left(\frac{1}{2} \frac{d r^{2}}{d x}\right)^{2}\right\}^{\frac{1}{2}} d x
\end{gathered}
$$

e per la formula (2):

$$
\sigma^{\prime}=2 \pi \int_{0}^{H}\left\{R^{2}(1+u)+\left(\frac{R^{2}}{2} \frac{d u}{d x}\right)^{2}\right\}^{\frac{1}{2}} d x
$$

ovrero:

$$
\sigma^{\prime}=2 \pi R \int_{0}^{H}\left\{1+u+\left(\frac{R}{2} \frac{d u}{d x}\right)^{2}\right\}^{\frac{1}{2}} d x
$$


Introduco la variabile:

$$
\theta=\frac{2 \pi}{H} x
$$

Potrò allora, per ogni valore del parametro $t$, considerare la $u$ come una funzione di $\theta$ definita fra $\theta=0$ e $\theta=2 \pi$. Per $\theta=0$ e $\theta=2 \pi$ sarà $u=0$. Sarà poi $d x=\frac{H}{2 \pi} d s$; onde la formula (3) diventerà

e la (4):

$$
\int_{0}^{2 \pi} u d \theta=0
$$

$$
\sigma^{\prime}=R H \int_{0}^{2 \pi}\left\{1+u+\left(\frac{\pi R}{H} \frac{d u}{d \theta}\right)^{2}\right\}^{\frac{1}{2}} d \theta .
$$

Più semplicemente, ponendo

scriveremo :

$$
q=\left(\frac{\pi R}{I I} \frac{d u}{d \theta}\right)^{2}
$$

$$
\left.\sigma^{\prime}=R H \int_{0}^{2 \pi} \mid 1+u+q\right\}^{\frac{1}{2}} d \theta
$$

Col tendere di $t$ a zero, $\frac{d u}{d \theta}$, che è uguale ad $\frac{H}{2 \pi} \frac{d u}{d x}$, tende uniformemente a zero, come $\frac{d u}{d x}$ : lo stesso avverrà di $q$, ed anche di $u+q$.

Ora osserviamo che

$$
(1+\varepsilon)^{\frac{1}{2}}=1+\frac{1}{2} \varepsilon-\frac{1}{8} \varepsilon^{2}\left(1+\varepsilon^{\prime}\right)
$$

ove $\varepsilon^{\prime}$ tende a zero con $\varepsilon$. Percio potremo scrivere:

$$
|1+u+q|^{\frac{1}{2}}=1+\frac{1}{2}(u+q)-\frac{1}{8}(u+q)^{2}\left(1+\varepsilon^{\prime}\right)
$$

ove $\varepsilon^{\prime}$ sarà una funzione di $\theta$ e $t$ che col tendere di $t$ a zero tende uniformemente a zero. 
Sviluppando $(u+q)^{2}$, e ponendo, per semplicità,

$$
\varepsilon^{\prime \prime}=-\left(\frac{1}{4} q+\frac{1}{2} u\right)\left(1+\varepsilon^{\prime}\right)
$$

avremo:

$$
|1+u+q|^{\frac{1}{2}}=1+\frac{1}{2} u-\frac{1}{8} u^{2}\left(1+\varepsilon^{\prime}\right)+\frac{1}{2} q\left(1+\varepsilon^{\prime \prime}\right) .
$$

Anche $\varepsilon^{\prime \prime}$ tende uniformemente a zero con $t$.

Sostituisco nella formula (6):

$$
\sigma^{\prime}=R H \int_{0}^{2 \pi}\left\{1+\frac{1}{2} u-\frac{1}{8} u^{2}\left(1+\varepsilon^{\prime}\right)+\frac{1}{2} q\left(1+\varepsilon^{\prime \prime}\right)\right\} d \theta .
$$

$\operatorname{Ma} R H \int_{0}^{2 \pi} d \theta=2 \pi R H=\sigma, \int_{0}^{2 \pi} u d \theta=0$. Dunque:

$$
\sigma^{\prime}=\sigma+\frac{R H}{8}\left\{4 \int_{0}^{2 \pi}\left(1+\varepsilon^{\prime \prime}\right) q d \theta-\int_{0}^{2 \pi}\left(1+\varepsilon^{\prime}\right) u^{2} d \theta\right\} .
$$

Osservando che $q$ ed $u^{2}$ sono quantità sempre positive o nulle, potremo scrivere:

$$
\sigma^{\prime}=\sigma+\frac{R \cdot H}{8}\left\{4\left(1+e^{\prime}\right) \int_{0}^{2 \pi} q d \theta-\left(1+e^{\prime \prime}\right) \int_{0}^{2 \pi} u^{2} d \theta\right\}
$$

ove $e^{\prime}$ ed $e^{\prime \prime}$ rappresenteranno dei valori medii di $\varepsilon^{\prime \prime}$ ed $\varepsilon^{\prime}$ fra 0 e $2 \pi$ : saranuo perciò funzioni del solo parametro $t$, che tendono a zero con $t$.

Ora torniamo a sostituire a $q$ il suo valore dato dalla formula (5). Avremo:

$$
\sigma^{\prime}=\sigma+\frac{R H}{8}\left\{\left(1+e^{\prime}\right)\left(\frac{2 \pi R}{H}\right)^{2} \int_{0}^{2 \pi}\left(\frac{d u}{d \theta}\right)^{2} d \theta-\left(1+e^{\prime \prime}\right) \int_{0}^{2 \pi} u^{2} d \theta\right\} .
$$

È questa l'espressione di $\sigma^{\prime}$ che volevamo stabilire.

4. Prima di andar più oltre esaminiamo un caso particolare. Sia $u=t \operatorname{sen} \theta$. Si riconosce immediatamente che questa funzione $u(\theta, t)$ sod- 
disfa a tutte le condizioni volute: infatti $u(0)=0, u(2 \pi)=0, \int_{0}^{2 \pi} u d \theta=0$, inoltre $u$ e $\frac{d u}{d !}$ sono continue, e tendono uniformemente a zern con $t$.

La formula (7), osservando che i due integrali del secondo membro sono, in questo caso, uguali a $\pi t^{2}$, diventerà:

$$
\sigma^{\prime}=\sigma+\frac{\pi R H t^{2}}{8}\left\{\left(1+e^{\prime}\right)\left(\frac{2 \pi R}{H}\right)^{2}-\left(1+e^{\prime \prime}\right)\right\} .
$$

Poichè $e^{\prime}$ ed $e^{\prime \prime}$ tendono a zero con $t$, la quantità entro le grandi parentesi tenderà al valore $\left(\frac{2 \pi R}{H}\right)^{2}-1$ : dunque, per $t$ abbastanza piccolo, sarà $\sigma^{\prime} \gtrless \sigma$ secondochè $H$ è minore o maggiore di $2 \pi R$.

Cos̀̀ intanto vediamo che quando $H$ è maggiore di $2 \pi R, \sigma^{\prime}$ può tendere verso $\sigma$ conservando, per valori abbastanza piccoli di $t$, un'area minore dell'area di $\sigma$.

5. Dopo eid ritorniamo al caso generale.

La funzione $u(\theta, t)$, per un determinato valore del parametro $t$, sarà una certa funzione $U(\theta)$, definita tra 0 e $2 \pi$, che soddisfa a queste condizioni:

1) è finita e continua insieme alla sua derivata $\frac{d U}{d \theta}$,

2) si annulla per $\theta=0$ e $\theta=2 \pi$,

3) verifica l'equazione $\int_{0}^{2 . \pi} U d \theta=0$.

Supponiamo di saper dimostrare che per qualunque funzione di tal natura si ha:

$$
\int_{0}^{2 \pi}\left(\frac{d U}{d \theta}\right)^{2} d \theta=\int_{0}^{2 \pi} U^{2} d \theta
$$

Sarà, per tutti i valori di $t$ :

$$
\int_{0}^{2 \cdot \pi}\left(\frac{d u}{d \theta}\right)^{2} d \theta=\int_{0}^{2 \pi} u^{2} d \theta
$$

Se dunque $H$ è minore di $2 \pi R$, esisterà un numero $t_{0}$ così piccolo, che 
per qualunque valore di $t$ compreso fra 0 e $t_{0}$ la quantità racchiusa dalle grandi parentesi, nella formula (7), sia positiva. Sarà allora $\sigma^{\prime}>\sigma$.

La questione, come si vede, è ridotta a dimostrare la formula (8).

6. Supponiamo da prima che la funzione $U$ sia rappresentata da una serie finita di Fođrrer : sia cioè :

$$
U=\sum_{i}^{N}\left\{a_{n} \operatorname{sen}(n \theta)+b_{n} \cos (n \theta)\right\}
$$

ove $N$ è un numero intero e positivo, $a_{n}$ e $b_{n}(n=1,2, \ldots, N)$ sono delle costanti. Ho tralaseiato il termine costante, dovendo essere $\int_{0}^{2 x} U d \theta=0$. Affinchè per $\theta=0$ ed $\theta=2 \pi$ sia $U=0$, basia supporre $\sum_{0}^{N} b_{n}=0: \operatorname{ma} \mathrm{di}$ questa condizione non occorrerà tener conto.

Dalla formula precedente si ha:

$$
U^{2}=\sum_{1}^{N}\left\{a_{n}^{2} \operatorname{sen}^{2}(n \theta)+b_{n}^{2} \cos ^{2}(n \theta)\right\}+\Sigma T
$$

ove $\Sigma T$ rappresenta una somma di termini, ciascuno dei quali, per proprietà ben note, verifica l'equazione:

Essendo poi

$$
\int_{0}^{2 \pi} T d \theta=0
$$

sarà

$$
\int_{0}^{2 \pi} \operatorname{sen}^{2}(n \theta) d \theta=\int_{0}^{2 \pi} \cos ^{2}(n \theta) d \theta=\pi
$$

$$
\int_{0}^{2 \pi} U^{2} d \theta=\pi \sum_{1}^{N}\left(a_{n}^{2}+b_{n}^{2}\right)
$$

Calcolando $\frac{d U}{d \theta}$ si troverà analogamente

$$
\int_{0}^{2 \pi}\left(\frac{d U}{d \theta}\right)^{2} d \theta=\pi \sum_{1}^{N} n^{2}\left(a_{n}^{2}+b_{n}^{2}\right)
$$


Dal confronto di questa formula colla precedente, la (8), per la classe speciale, ma pur vastissima, di funzioni $U$ che consideriamo, resulta senz'altro verificata.

7. Sia ora $U$ una funzione qualunque che soddisfi alle condizioni 1 ), $2), 3)$. Inoltre supponiamo che la sua derivata $\frac{d U}{d \theta}$ assuma gli stessi valori per $\theta=0$ e $\theta=2 \pi$.

Allora, per un Teorema di Analisi dovuto al PiCard (*), potremo rappresentare la funzione $\frac{d U}{d \theta}$ con $U^{\prime}+\lambda$, ove $U^{\prime}$ è una serie finita del Fourier, e $\lambda$ una funzione di $\theta$, la quale, prendendo abbastanza grande il numero di termini di $U^{\prime}$, può rendersi, in tutto l'intervallo fra 0 e $2 \pi$, minore, in valore assoluto, di un numero assegnato, piccolo ad arbitrio.

Evidentemente potremo porre $U^{\prime}=\frac{d U_{1}}{d \theta}+c, c$ essendo il termine costante di $U^{\prime}$, ed $U_{1}$ un'altra serie finita del Fourien, il cui termine costante si puo supporre nullo. Avremo percio

$$
\frac{d U}{d \theta}=\frac{d U_{1}}{d \theta}+c+\lambda
$$

Moltiplico per $d \theta$ e integro fra 0 e $2 \pi$. Poichè $U(2 \pi)=U(0), U_{1}(2 \pi)=U_{1}(0)$, otterro, risolvendo rispetto a $c$ :

$$
c=-\frac{1}{2 \pi} \int_{0}^{2 \pi} \lambda d \theta \text {. }
$$

Questa formula mostra che la costante $c$ può rendersi, in valore assoluto, piccola ad arbitrio. Pongasi allora $c+\lambda=\mu$ : avremo:

$$
\frac{d U}{d \theta}=\frac{d U_{1}}{d \theta}+\mu
$$

ove $\mu$ è una funzione che potremo rendere, in valore assoluto, piccola ad arbitrio, in tutto l'intervallo fra 0 e $2 \pi$.

(*) Traité d'Analyse, Tome I, p. 257. 
Integrando fra 0 e $\theta$ si ottiene:

ove $\mu_{1}=\int_{0}^{\theta} \mu d s$ e $c_{1}$ è una nuova costante.

$$
U=U_{1}+\mu_{1}+c_{1}
$$

Poichè $\int_{0}^{2 \pi x} U d \theta=\int_{0}^{2 \pi} U_{1} d \theta=0$, dovrà essere $c_{1}=-\frac{1}{2 \pi} \int_{0}^{2 \pi} r_{1} d s$. Ponendo

$\mu_{1}+c_{1}=\nu$, avremo:

$$
U=U_{1}+\nu
$$

ove $\nu$ è una funzione che al pari di $\mu$ e $\mu_{1}$ si può rendere, in valore assoJuto, piccola ad arbitrio, in tutto l'intervallo fra 0 e $2 \pi$.

Dalle formole (10) e (11) si riconosce che i due integrali $\int_{0}^{2 \pi} U_{1}^{2} d \theta$, $\int_{0}^{2 / \pi}\left(\frac{d U_{1}}{d \theta}\right)^{2} d \theta$ si possono far differire di tanto poco quanto si vuole dagl'integrali analoghi formati colla funzione $U$. Ma per la funzione $U_{1}$, che è una serie finita del Fourier senza termine costante, vale una formula analoga alla (8): dunque per la funzione $U$ varrà la formula (8).

8. Ora, finalmente, sia $U$ una funzione che soddisfi alle sole condizioni 1), 2), 3): la sua derivata $\frac{d U}{d A}$ non assumerà, in generale, valori uguali, per $\theta=0$ e $\theta=2 \pi$.

È facile vedere che si può sempre costruire una funzione $U^{\prime}$ che soddisf, come la $U$, alle condizioni 1$), 2$ ), 3), la cui derivata $\frac{d U^{\prime}}{d \theta}$ assuma valori uguali per $\theta=0$ e $\theta=2 \pi$, e sia tale che $\mathrm{i}$ due integrali $\int_{0}^{2 \pi} U^{\prime 2} d \theta$ $\int_{0}^{2 \pi}\left(\frac{d U^{\prime}}{d \theta}\right)^{2} d \theta$ differiscano di tanto poco quanto si vuole dagl'integrali analoghi formati colla funzione $U$. Ma per la funzione $U^{\prime}$ vale una formula analoga 
alla (8) (§ 7): questa formula dovrà dunque essere verificata anche dalla funzione $U$.

Il Teorema pertanto è dimostrato.

9. Si noti che nei parayrafi precedenti non abbiamo tenuto conto della condizione $U(0)=0, U(2 \pi)=0$, ma solo della condizione meno restrittiva $U(0)=U(2 \pi)$, la quale potrà perciò esser sostituita alla 2$)$.

Così la formola (9) varrà quand'anche $u(0)$ e $u(2 \pi)$ non siano uguali a zero, purchè, per qnalunque valore di $t$, siano uguali tra loro.

Ciò porta come conseguenza che il teorema dimostrato $\left(\sigma^{\prime}<\tau\right.$, se $H<2 \pi R$ ) sussiste anche se, col variare di $t$, le sezioni estreme di $\sigma^{\prime}$ variano, purchè l'area dell'una sia sempre uguale all'area dell'altra.

Se però le due aree non sono uguali tra loro, potrà accadere che, pur essendo $I T<2 \pi R$, la superficie $\sigma^{\prime}$, col tendere di $t$ a zero, si conservi sempre minore di $\sigma$.

Consideriamo infatti una speciale superficie $\sigma^{\prime}$, di rivoluzione, definita dalla formula $r^{2}=R^{2}(1+u)(\S 2)$, ove

$$
u=t \mid-\pi \cos \theta+\theta-\pi\} \text {. }
$$

La condizione $\int_{0}^{2 . x} u d \theta$ è soddisfatta: dunque il volume racchiuso da questa superficie è sempre uguale a $\pi R^{2} H$. Ma le due sezioni estreme non sono uguali: infatti per $\theta=0$ abbiamo $u=-2 \pi t$, quindi $\pi r^{2}=\pi R^{2}(1-2 \pi t)$; per $\theta=2 \pi, u=0$ e $\pi r^{2}=\pi R^{2}$. Le due aree differiscono di $2 \pi^{2} R^{2} t$.

La formula (7) sussiste indipendentemente dai valori che assume $u$ per $\theta=0$ e $\theta=2 \pi$. Calcolando i due integrali che vi figurano, troveremo:

quindi :

$$
\int_{0}^{2 x} u^{2} d \theta=\left(\pi+\frac{2}{3} \pi^{3}\right) t^{2}, \quad \int_{0}^{2 \mu}\left(\frac{d u}{d \theta}\right)^{2} d \theta=3 \pi t^{2}
$$

$$
\sigma^{\prime}=\sigma+\frac{\pi R H t^{2}}{8}\left\{3\left(1+e^{\prime}\right)\left(\frac{2 \pi R}{H}\right)^{2}-\left(1+\frac{2}{3} \pi^{2}\right)\left(1+e^{\prime \prime}\right)\right\} .
$$

La quantità entro le grandi parentesi, col tendere di $t$, e per conseguenza di $e^{\prime}$ ed $e^{\prime \prime}$, a zero, tende al valore

$$
3\left(\frac{2 \pi R}{H}\right)^{2}-\left(1+\frac{2}{3} \pi^{2}\right)
$$


che può esser negativo se $H$ è minore di $2 \pi R$, giacchè $1+\frac{2}{3} \pi^{2}$ è maggiore di 3 .

In tal caso, per $t$ abbastanza piccolo, $\sigma^{\prime}$ è minore di $\sigma$.

Qualunque sia il valore del rapporto $\frac{H}{2 \pi R}$, la formula (12), indicando con a la differenza $2 \pi^{2} R^{z} t$ fra le sezioni estreme di $\sigma^{\prime}$, si potrà scrivere:

$$
\sigma^{\prime}-\sigma=Q a^{2}
$$

ove $Q$ è una quantità che si conserva sempre finita.

Più avanti ci sarà utile questo resultato.

10. Noi vugliamo ora dimostrare un teorema più generale di quello di cui abbiamo dato la dimostrazione nei $\S \S 2-8$ : un teorema, cioè, relativo ad una superficie qualunque $\sigma^{\prime}$, limitata da due

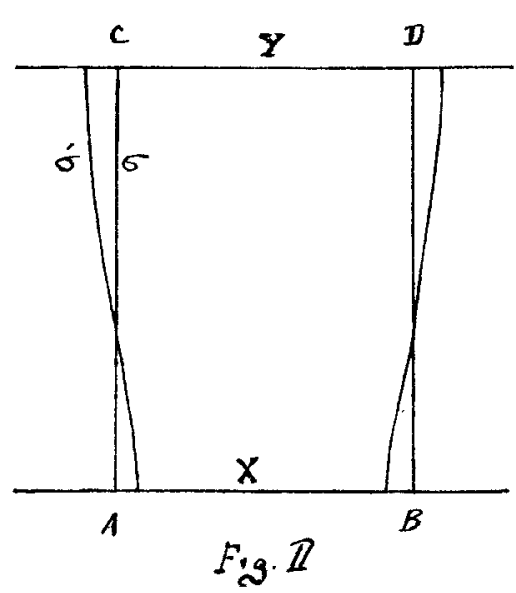
piani paralleli $X, Y$, (Fig. 2), la quale, col tendere a zero di un parametro $t$, tenda verso la superficie cilindrica $\sigma$ di raggio $R$, limitata dagli stessi piani, togliendo le condizioni che le sue sezioni estreme siano uguali tra loro, e che essa racchiuda un volume uguale a $\pi R^{2}$.

La distanza $H$ fra i due piani $X, Y$ sia minore di $2 \pi R$.

Il nostro scopo è quello di stabilire un limite inferiore di $\sigma^{\prime}$, tenendo conto del volume che essa racchiude, e della differenza fra l'area delle sezioni estreme. Percio potremo supporre che $\sigma^{\prime}$ sia una superficie di rivoluzione $(\$ 2)$.

Sia $\pi R^{\prime 2} H$ il volume racchiuso da $\sigma^{\prime}, R^{\prime}$ denotando una quantità che col tendere di $t$ a zero tende verso $R$.

Invece della formula (2) si potrà scrivere:

$$
r^{2}=R^{\prime 2}(1+v)
$$

$v$ essendo una funzione di $\theta$ e di $t$ che tende uniformemente a zero con $t$, insieme alla sua derivata $\frac{d v}{d \dot{\theta}}$, e soddisfa alla equazione $\int_{0}^{2 \pi} v d \theta=0$, ma può non assumere valori uguali per $\theta=0$ e $\theta=2 \pi$. 
Invece della formula (7), ove $\sigma$ vale $2 \pi R H$, avremo:

$$
\begin{gathered}
\sigma^{\prime}=2 \pi R^{\prime} H+ \\
+\frac{R^{\prime} H}{8}\left\{\left(1+e^{\prime}\right)\left(\frac{2 \pi R^{\prime}}{H}\right)^{2} \int_{0}^{2 \pi}\left(\frac{d v}{d \theta}\right)^{2} d \theta-\left(1+e^{\prime \prime}\right) \int_{0}^{2 \pi} v^{2} d \theta\right\} .
\end{gathered}
$$

Siano $S_{1}$ ed $S_{2}^{\prime}$ le sezioni estreme della superficie $\sigma^{\prime}$, vale a dire, per la formula (13):

$$
S_{1}=\pi R^{2}\{1+v(0)\}, \quad S_{2}=\pi R^{\prime 2}\{1+v(2 \pi)\} .
$$

Poniamo

ovvero

$$
a=S_{z}-S_{1},
$$

$$
a=\pi R^{\prime 2}\{v(2 \pi)-v(0)\} \text {. }
$$

La quantità a tende a zero con $t$.

Consideriamo la nuova funzione di $\sigma$ e $t$ :

$$
u=v-\frac{a}{2 \pi^{2} R^{\prime 2}}(\theta-\pi)
$$

Essa soddisfa la condizione $\int_{0}^{2 \pi} u d \theta=0$, ed assume valori uguali per $\theta=0 \mathrm{e}$ $\dot{\theta}=2 \pi$

Sostituendo, nella formula (14), $u+\frac{a}{2 \pi^{2} R^{\prime 2}}(\xi-\pi)$ a $v$, e $\frac{d u}{d \theta}+\frac{a}{2 \pi^{2} R^{\prime 2}}$ a $\frac{d v}{d t}$, otterremo:

$$
\sigma^{\prime}=2 \pi R^{\prime} H+\frac{R^{\prime} H}{8}\left\{\left(1+e^{\prime}\right)\left(\frac{2 \pi R^{\prime}}{H}\right)^{2} \int_{0}^{2 \pi}\left(\frac{d u}{d \theta}\right)^{2} d \theta-\left(1+e^{\prime \prime}\right) \int_{0}^{2 x} u^{2} d \theta\right\}+g a,
$$

ove $g$ è la somma di più termini che contengono come fattori quantità infinitesime, e quindi tende a zero con $t$.

Se H è minore di $2 \pi R$, per $t$ abbastanza piccolo sarà anche minore di $2 \pi R^{\prime}$, giacchè $R^{\prime}$ tende verso $R$. Inoltre il primo dei due integrali che figurano nella formula precedente è maggiore del secondo od uguale (formula $(9)$ ). Sarà dunque, per $t$ abbastanza piccolo:

$$
\sigma^{\prime}>2 \pi R^{\prime} H+g a,
$$


ovvero:

$$
\sigma^{\prime}-\sigma>2 \pi H\left(R^{\prime}-R\right)+g a \quad(\sigma=2 \pi R H)
$$

Ma $2 \pi H\left(R^{\prime}-R\right)=\frac{2}{R+R^{\prime}}\left|\pi R^{\prime 2} H-\pi R^{2} H\right|=-G w, w$ rappresentando la differenza $\pi R^{2} H-\pi R^{2} H$ fra il volume racchiuso dalla superficie $\sigma$, e quello racchiuso dalla superficie $\sigma^{\prime}$, e $G$ una quantita sempre finita. Arremo peroid

$$
\sigma^{\prime}-\sigma>-G v+g a .
$$

Questa formula esprime il teorema che volevamo dimostrare.

11. Torniamo ora a considerare la goccia d'olio in equilibrio sotto la forma di una colonna cilindrica d'altezza $H$, li-

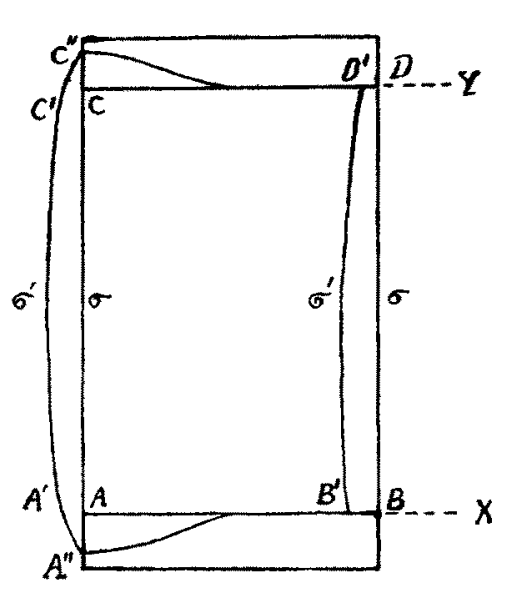

Fig. III mitata dai bordi $A B, C D$ dei due dischi di raggio $R$ (Fig. 3 ).

Diamole una deformazione infinitesima, in modo che essa lasci scoperta complessivamente una certa porzione a $\left(B B^{\prime}+D D^{\prime}\right)$ dei cerchi $A B, C D$, e venga a coprire una porzione $y$ $\left(\boldsymbol{A} \boldsymbol{A}^{\prime \prime}+C C^{\prime \prime}\right)$ della superficie laterale dei dischi.

Indichiamo con $\sigma^{\prime}+\lambda$ la nuova superficie di separazione fra l'olio e il liquido circostante, intendendo che $\sigma^{\prime}$ rappresenti quella parte della superficie che è compresa fra i due piani $X, Y$ in cui si trovano i cerchi $A B, C D$.

Si suppone che $\alpha, \gamma$ e $\lambda$ tendano a zero, e $\sigma$ tenda verso $\sigma$, col tendere a zero di un parametro $t$.

Consideriamo la variazione $\Delta W=m \Delta \sigma-n \Delta \tau(\S 1)$ del potenziale $W$. Sarà evidentemente :

quindi

$$
\Delta \sigma=\sigma^{\prime}+\lambda-\sigma, \Delta==-\alpha+\%
$$

$$
\Delta W=m\left(\sigma^{\prime}+\lambda-\sigma\right)-n(-\alpha+\gamma)
$$

ovvero:

$$
\Delta W=m\left(\sigma^{\prime}-\sigma\right)+m \lambda+n \sigma-n \gamma
$$


In particolare possiamo supporre che la goccia d'olio deformata non esca fuori dei piani $X, Y$. Sarà allora $\gamma=\lambda=0$, e

$$
\Delta W=m\left(\sigma^{\prime}-\sigma\right)+n \alpha .
$$

Se $H$ è maggiore di $2 \pi R$, supponendo $\alpha=0, \sigma^{\prime}<\sigma(\S 4)$, sarà $\Delta W<0$. La variazione di $W$ può dunque esser negativa.

Sia invece $H<2 \pi R$. Esaminiamo da prima una deformazione speciale: la superficie $\sigma^{\prime}$ sia quella considerata nel $\$ 9$. Si è trovato

$$
\sigma^{\prime}-\sigma=Q a^{2}
$$

ove. $Q$ è una quantità finita, ed a rappresenta la differenza fra $\pi R^{2}$ ed una delle sezioni estreme di $\sigma^{\prime}$ : l'altra era uguale a $\pi R^{z}$. Dunque $a$ non è altro che $\alpha$; e potremo scrivere (poichè in questo caso $y=\lambda=0$ ):

$$
\Delta W=m Q \alpha^{2}+n \alpha=(n+m Q \alpha) \alpha .
$$

Affinchè possa aversi l'equilibrio, stabile o instabile, ̀̀ necessurio che sia $\Delta W \equiv 0$, quindi $n+m Q \propto \equiv 0$, ovvero, $\propto$ essendo una quantita infinitesima,

$$
n \equiv 0 \text {. }
$$

Consideriamo ancora una deformazione particolare: $\alpha, \sigma^{\prime}-\sigma$ e $\lambda-\gamma$ siano infinitesime d'ordine superiore rispetto a $\gamma$. Ciò è possibile, giacchè per un dato valore di $\gamma$, possiamo rendere $\alpha$ e $\sigma^{\prime}-\sigma$ piccole ad arbitrio, e $\lambda$ vicino a $\gamma$ quanto si vuole.

Avremo allora, per la formula (16):

$$
\Delta W=(m-n) \gamma+\varepsilon,
$$

ove $\varepsilon$ è una quantità infinitesima d'ordine superiore rispetto a $\gamma$.

Per l'equilibrio dovrà essere $m-n \geqslant 0$.

Noi escluderemo il caso che sia esattamente $n=0$ od $n=m$. Dovremo supporre pertanto

$$
0<n<m \text {. }
$$

12. Riprendiamo la formula generale (16). Pongasi $m=n+2 p(p>0)$. Sarà $m \grave{\lambda}=n \lambda+2 p \lambda$, quindi

$$
\Delta W=m\left(\sigma^{\prime}-\sigma\right)+n(\lambda-\gamma)+2 p \lambda+n \alpha ;
$$

e poichè $\lambda=\gamma$ ( $\gamma$ essendo la proiezione sul cilindro di raggio $R$ della super- 
ficie esterna $\lambda)$ :

$$
\Delta W=m\left(\sigma^{\prime}-\sigma\right)+2 p \lambda+n \alpha .
$$

Diciamo $\beta$ la proiezione $\left(A A^{\prime}+C C^{\prime}\right)$ di $\lambda$ sui piani $X, Y$. Sarà $\lambda \equiv \beta, 2 p \lambda \equiv p \lambda+p \beta$, onde

$$
\Delta W \equiv m\left(\sigma^{\prime}-\sigma\right)+p \lambda+p \beta+n \alpha,
$$

e chiamando $q$ la minore delle due quantità $p$ ed $n$ :

$$
\Delta W=m\left(\sigma^{\prime}-\sigma\right)+p \lambda+q(\alpha+\beta) \text {. }
$$

Ora teniamo conto della formula (15). In essa $w$ rappresenterà il volume di quella parte della goccia d'olio che si trova fuori dei piani $X, Y$ : a è la differenza fra le sezioni estreme di $\sigma$. Avremo:

$$
\Delta W>-m G w+m g a+p \lambda+q(\alpha+\beta),
$$

ovvero:

$$
\Delta W>|p \lambda-m G w|+|q(\alpha+\beta)+m g a| \text {. }
$$

Il volume $w$ è racchiuso dalla superficie $\beta+\gamma+\lambda \equiv 3 \lambda$. Il massimo volume che può racchiudere una superficie uguale a $3 \lambda$ è $\frac{2 \sqrt{\pi}}{\gamma^{3}} \lambda^{\frac{3}{2}}$ (caso della sfera). Dunque $w$ è infinitesimo d'ordine superiore rispetto a $\lambda$. D'altra parte $p, m$ e $G$ sono quantità finite, e $p$ è positiva. Quindi sarà, per $t$ abbastanza piccolo, $p \lambda-m G w \equiv 0$.

Ora consideriamo le sezioni estreme della superficie $\sigma^{\prime}\left(A^{\prime} B^{\prime}+C^{\prime} D^{\prime}\right)$. Ciascuna di esse è uguale a $\pi R^{2}$ - una parte di $\alpha+$ una parte di $\beta$ : ̀̀ dunque compresa fra $\pi R^{2}-\alpha$ e $\pi R^{2}+\beta$. La loro differenza $a$, in valore assoluto, sarà minore di $\alpha+\beta$. Poichè $q$ ed $m$ sono quantità finite mentre $g$ è infinitesima $(\S 10)$, per $t$ abbastanza piccolo sarà

E per conseguenza:

$$
q(\alpha+\beta)+m g a \equiv 0 \text {. }
$$

$$
\Delta W>0,
$$

cio che appunto volevamo dimostrare.

13. Il potenziale $W=m \sigma-n \tau$ si riferisce ad un sistema costituito dei sostegni rigidi e dei due liquidi.

Supponiamo di scambiare tra loro $\mathrm{i}$ due liquidi, e cerchiamo il nuovo potenziale $W^{\prime}$, analogo a $W$. 
Indicando con $++\tau$ la superficie totale dei sostegni rigidi, basterà sostituire $\tau$ con $\tau$; avremo dunque:

$$
W^{\prime}=m \sigma-n \tau^{\prime},
$$

orvero, a meno di una costante:

$$
W^{\prime}=m \tau+n \tau ; \quad\left(\tau+\tau^{\prime}=\text { cost. }\right)
$$

vale a dire: passando da un sistema all'altro il coefficiente di $\sigma$ non varia, quello di $\tau$ cambia di segno.

Ma per l'equilibrio del primo sistema si è veduto che deve essere $n \equiv 0$. Per l'equilibrio del secondo si dovrà dunque avere $n \equiv 0$.

Tali condizioni non sono compatibili tra loro se non quando sia $n=0$. Fatta dunque eccezione da questo caso speciale, possiamo dire che se è possibile l'equilibrio di una colonna cilindrica costituita di un certo liquido $L$, immersa in un altro liquido $L$ ', non è possibile l'equilibrio del sistema ottenuto invertendo $\mathrm{i}$ due liquidi.

L'annullarsi di $n$ ha un significato fisico assai semplice. Supponiamo che la superficie dei sostegni, lungo la linea d'incontro colla superficie $\sigma$ che separa i due liquidi, ammetta un piano tangente unjco (condizione che non è verificata nel caso dei dischi): se $n$ è uguale a zero, le due superficie s'incontrano normalmente, giacchè, detto $\varphi$ l'angolo di raccordo, si ha dalla Teoria di Gauss

$$
\cos \varphi=\frac{n}{m}
$$

